Eine Betrachtung der Isotopentabelle zeigt ferner, daß keine Möglichkeit besteht, etwa ein KEinfang zeigendes Hf-Isotop für die 19-Sek.-Aktivität verantwortlich zu machen, da entsprechende Cp-Isotope fehlen bzw. als Folgeprodukte durch ihre Aktivität auffallen müßten.

Es muß daher geschlossen werden, daß es sich bei der Aktivierung des kurzlebigen Körpers um einen (n, $\gamma$ )-Prozeß eines Hf-Isotops handelt, der zum nächsthöheren stabilen Isotop führt und dieses in einen angeregten Zustand versetzt, dessen Anregungsenergie $250(190+65) \mathrm{KeV}$ beträgt und der mit einer Halbwertszeit von 19 Sek. in den Grundzustand übergeht. Zur Frage der Zuordnung zu einer Massenzahl müssen wir wieder die Mattauchsche Kernisomeren-Regel heranziehen. Die Massenzahl 174 soll wegen der geringen Häufigkeit des
Isotops außer Betracht bleiben. Wegen der Matt a uch schen Regel sind die Kerne 176, 178, 180, die sämtlich dem Typus g-g angehören, auszuschließen, so daß nur 177 und 179 übrig bleiben. Da beide gleicher Häufigkeit sind, können wir keine Wahl zwischen ihnen treffen. Wir wollen daher die beobachtete Aktivität von 19 Sek. versuchsweise dem ${ }^{177},{ }^{179} \mathrm{Hf*}$ zuordnen.

Für beide Isotope liegen auch Angaben über den Kernspin vor ${ }^{13}$. Er beträgt bei beiden $\leqslant 3 / 2$. Sollte der Kernspin $i=3 / 2$ zutreffen, so wäre dies das erstemal, da\& für einen solchen Fall ein Isomer existierte, während der Spin $i=1 / 2$ ausgezeichnet zu den anderen kernphysikalischen Erfahrungen passen würde.

${ }^{13}$ E. Rasmussen, Naturwiss. 23, 69 [1935].

\title{
Quantentheorie der Feldmechanik
}

\author{
Von FrITZ Bopp \\ Aus dem Kaiser-Wilhelm-Institut für Physik, Hechingen \\ (Z. Naturforschg. 1, 196-203 [1946]; eingegangen am 2. März 1946)
}

\begin{abstract}
Die früher abgeleiteten Bewegungsgleichungen der Feldmechanik werden - zunächst ohne Berücksichtigung der Strahlungskraft - nach dem Retardierungsparameter entwickelt, und zwar einen Schritt weiter als in der L or en tz schen Theorie des Elektrons. Die resultierenden Bewegungsgleichungen enthalten neue Freiheitsgrade, die eine Zitterbewegung beschreiben und bereits im klassischen Bereich zu spinartigen Drehimpulszusätzen Anlaß geben. Der formale Übergang zur Quantenmechanik macht keine Schwierigkeit und führt zu einer Wellengleichung vom Diracschen Typ allerdings derart, daß zunächst nur I.ösungen mit ganzzahligem Spin möglich sind und daß im allgemeinen eine starke Kopplung besteht zwischen Lösungen, die zu verschiedenem Spin gehören. Der Spezialfall des kräfte- und impulsfreien Teilchens wird besonders untersucht. Die Wellengleichung vereinfacht sich, wird aber keineswegs trivial. Ihre Eigenlösungen geben $\mathrm{Zu}-$ stände des Elementarteilchens, die zu verschiedenen Massen- und Spinwerten gehören, fassen also prinzipiell die Gesamtheit der Elementarteilchen in einer Wellengleichung zusammen. Quantitativ sind die Ergebnisse in der vorliegenden Näherung noch unbefriedigend. Die Art der Abweichung von den wirklichen Verhältnissen liegt nach früheren Untersuchungen an der Näherung und ist von vornherein zu erwarten.
\end{abstract}

\section{§1. Zur Quantelung der Feldmechanik}

$D_{\text {in }}^{\text {en }}$ V Versuch, die Singularitätenschwierigkeit in der Quantentheorie der Wellenfelder durch Abänderung der Maxwellschen Gleichungen zu lösen, begegnet man im allgemeinen mit Vorbehalt. Es ist die Meinung verbreitet, daß die Annahme spezieller Struktureigenschaften unterhalb endlicher Grenzdimensionen ebenso sinnlos sei wie die von gleichzeitigen Orts- und Impulsmessungen, deren Genauigkeit die Heisenbergsche Unschärferelation übersteigt. C. F. Weizsäcker ${ }^{1}$ glaubt sogar, daß man die Notwendigkeit einer solchen Annahme mindestens in speziellen Fällen aus der Unschärferelation ableiten kann. Er zeigt, daß

1 ,Theorie des Mesons“ in Heisenbergs ,Vorträge über kosmische Strahlung“, Springer 1943, 10, 7, S. 108. 
es nicht möglich ist, die aus Konvergenzgründen notwendigen Abweichungen des $\mathrm{Y}$ u k a w a - Feldes von der kanonischen Gestalt $e^{-\varkappa \mathrm{r}} / r$ durch Streuexperimente festzustellen.

Gleichwohl werden wir im folgenden versuchen, aùs der früher entwickelten klassischen Feldmechanik $^{2}$ quantentheoretische Folgerungen $\mathrm{zu}$ ziehen. Vorliegende Ansätze für eine „Überquantenmechanik" ${ }^{\text {"3 }}$ sind kaum so weit gefördert, daß sie deren Notwendigkeit beweisen. Die bisherigen Formulierungen scheinen mir außerdem nicht ohne Strukturaussagen auszukommen. In der Quantengeometrie von-M arch ist es die Frage nach der Wahrscheinlichkeit, daß zwei Punkte noch unterscheidbar seien. Bei Heisenberg muß man eine Aussage darüber erwarten, wie die $\eta$-Matrix zweier benachbarter Streuzentren aussieht, wenn sie für das einfache Streuzentrum bekannt ist. Auch wenn man die Ortsunbestimmtheit durch Vertauschungsrelationen erfassen will, scheint mir die Einführung von Strukturfunktionen notwendig.

Die quantentheoretischen Argumente v. Weizsäckers schließen m. E. die Möglichkeit von Strukturaussagen nicht aus. Man denke sich das von v. Weizsäcker diskutierte Teilchen aus zwei sehr schweren Komponenten zusammengesetzt, deren Bindung so stark sei, daß der Massendefekt die beiden Teilmassen fast vollständig kompensiert. Für das Gesamtgebilde ist zweifellos die v. Weizsäckersche Behauptung richtig. Doch muß es möglich sein, über die gegenseitige Lage der Komponenten um so genauere Aussagen zu machen, je größer deren Masse ist. Die Quantentheorie läßt also Strukturaussagen $\mathrm{zu}$, ohne daß diese durch Streuexperimente nachweisbar sein müssen. Sie spiegeln sich wider, wie sich zeigen wird, in der Mannigfaltigkeit der möglichen Energiezustände des Teilchens bzw. in der Gesamtheit der in der Natur vorkommenden Elementarteilchen.

Selbstverständlich soll obiges Bild nur die Tragweite des v. Weizsäcker schen Schlusses deutlich machen, nach welchem bereits quantentheoretische Überlegungen Grenzen für die Anwendbarkeit der Quantentheorie zeigen sollten. Es soll

2 F. B opp, I, Ann. Physik (5) 38, 549 [1940]; II, Ann. Physik (5) 42, 572 [1943] ; III, Ann. Physik (einges. Juli 1944, Ersch. ungewiß); IV, Physik. Z. (einges. Dez. 1944, Kurzmitt. Jan. 1945, Korr. März 1945); V, Z. Natuxforschg. 1, 53 [1946].

3 A. M a r ch, Naturwiss. 31, 49 [1943]; W. H e i s e n ber g,, I, Z. Physik 120, 513 [1943] ; II, Z. Physik 120, 673 [1943]; III, Z. Physik 176, - [1944]; IV, Z. Physik; für die Überlassung eines Skriptums danke ich herzlich. weder eine ad hoc eingeführte Hypothese über die Struktur des Elektrons sein noch ein Beweis dafür, daß die bisherigeQuantenmechanik für die Theorie der Elementarteilchen ausreicht. Die Entscheidung darüber liefert allein der Vergleich der vollendeten Theorie mit der Erfahrung.

Den Ausgangspunkt unserer Untersuchung bildet die klassische Feldmechanik (1. c. 2, IV und V). Die Bewegungsgleichungen der Feldmechanik folgen aus den Wellengleichungen für die Feldgrößen unter der Voraussetzung, daß sich die felderzeugenden Teilchen im Gesamtfeld, d. h. im Eigenfelde und im Felde der übrigen Teilchen kräftefrei bewegen. Im Falle vektorieller Felder ist der Anteil des Außenfeldes die L o r e n t z-Kraft, der des Eigenfeldes zerfällt in Trägheits- und Strahlungskraft. Die Quantelung der Bewegungsgleichungen der Feldmechanik kann gemäß ihrer Herkunft grundsätzlich auf zwei Wegen erfolgen. Erstens kann man den Übergang zur Bewegungsgleichung nach der Quantelung des Wellenfeldes durchführen, wie es $\mathrm{Pais}{ }^{4}$ versucht hat. Da die bisherige Theorie der Wellenfelder an die duale Theorie für Feld und Teilchen anknüpft, bietet die Quantelung der feldbestimmten Trägheitskraft grundsätzliche Schwierigkeiten. Der Ausweg von Pais, gleichsam dual zu rechnen unter der Annahme eines verschwindenden Massengliedes in der Gleichung für Materiewellen, liefert wahrscheinlich eine praktisch ausreichende Genauigkeit, kann aber nicht völlig befriedigen, weil der Ansatz für die Materiewellen zusätzliche Annahmen erfordert ohne klassische Korrespondenz. Zweitens kann man den Übergang zur Bewegungsgleichung noch im klassischen Bereich vollziehen, um aus dieser die Quantenmechanik zu entwickeln. Hierbei macht umgekehrt die Strahlungskraft wegen ihres energiezerstreuenden Charakters Schwierigkeiten.

Eine willkürfreie Lösung findet man m. E. nur dann, wenn man beide Methoden etwa so kombiniert, wie es in der dualen Theorie geschieht. Man gehe von den Bewegungsgleichungen aus unter Weglassung der Strahlungskraft. 'Diese vereinfachten Gleichungen lassen sich aus einem $\mathrm{Ha}$ milto nschen Prinzip ableiten, dessen LagrangeFunktionstyp bereits M. Born ${ }^{5}$ angegeben hat

${ }^{4}$ A. Pais, On the Selfenergy of the Electron; Verf. danke ich für die Übersendung eines Skriptums durch Hrn. Prof. R os enfeld. Über die Veröffentlichung ist mir unter den gegenwärtigen Umständen nichts mehr bekannt gew orden.

${ }_{5}$ Proc. Roy. Soc. [London] Ser. A 143, 410 [1933/34]. 
(vergl. auch 1. c. ${ }^{2}$, I § 2). Ihre Quantelung bereitet keine grundsätzlichen Schwierigkeiten. Die Berücksichtigung der Ausstrahlung kann anschliefend erfolgen wie in der gewöhnlichen Strahlungstheorie. Die Vorwegnahme der Bewegungsgleichungen ohne Strahlungskraft hat in der Quantentheorie im Gegensatz zur klassischen Mechanik wegen der Existenz stationärer Zustände einen guten Sinn.

$\S 2$. Wellengleichung der Feldmechanik

Im folgenden beschränken wir uns darauf, den ersten Teil der eben formulierten Aufgabe durchzuführen. Wir wollen die zum reversiblen Teil der Bewegungsgleichung der Feldmechanik gehörige Wellengleichung ableiten, dabei abèr nur die ersten Schritte einer Entwicklung nach dem Retardierungsparameter berücksichtigen. In nullter Näherung erhält man die bekannten Bewegungsgleichungen, aus denen die K l ei in - G o r d o n sche Gleichung folgt. Die Gleichungen erster Näherung sollen hier berechnet und untersucht werden.

Das Wirkungsintegral für ein Teilchen mit den Koordinaten $x_{a}=x_{a}(s), u_{\alpha}=\dot{x}_{a}(s)(\alpha=1 \ldots 4)$ in einem äußeren Felde (Viererpotential $A_{\varrho}$ ) hat folgende $\mathrm{Form}^{6}$ :

$$
\begin{aligned}
\int \mathcal{L} d s=\frac{e^{2}}{c^{3}} \int u_{a}(s) u_{a}\left(s^{\prime}\right) & f(\sigma) d s d s^{\prime} \\
& -\frac{e}{c} \int u_{c} A_{c} d s .
\end{aligned}
$$

Darin ist

$$
\sigma=\frac{1}{c^{2}}\left[x_{c}(s)-x_{\alpha}\left(s^{\prime}\right)\right]^{2} .
$$

Die Lag r ange-Funktion lautet also

$$
\mathcal{L}=\frac{e^{2}}{c^{3}} \int u_{a}(s) u_{c}(s-\tau) f(\sigma) d \tau-\frac{e}{c} u_{\alpha} A_{\alpha},
$$

wobei $\sigma$ aus Gl. (2) hervorgeht, wenn man $s^{\prime}$ durch $s-\tau$ ersetzt. Die Funktion $f(\sigma)$ charakterisiert die spezielle Art der Feldgleichungen; z. B. ist für die Maxwell schen ${ }^{7}$ Gleichungen $f(\sigma)=\delta(\sigma)$. Allgemein gilt: $\int f(\sigma) \delta \sigma=1$.

Die Entwicklung nach dem Retardierungsparameter ergibt in erster Näherung

$$
\begin{gathered}
\mathcal{L}=-m c \sqrt{-u_{\alpha}^{\chi}}-\frac{e}{c} u_{\alpha} A_{\alpha}, \\
m=\frac{e^{2}}{c^{3}} \int f\left(-\tau^{2}\right) d \tau,
\end{gathered}
$$

woraus die bekannten Bewegungsgleichungen (für negative Ladungen) folgen:

$$
\frac{d}{d s} \frac{m u_{\alpha}}{\sqrt{-u_{\gamma / c^{2}}^{2}}}=-\frac{e}{c} f_{\alpha \beta} u_{\beta} .
$$

Das Wirkungsintegral in Gl. (1) ist ebenso wie das aus Gl. (4) folgende parameterinvariant. Es gilt also in gleicher Form für beliebige Parameter. Dies garantiert, daß man dafür z. B. die Eigenzeit wählen kann, daß also die Nebenbedingung $u_{c^{2}}=-c^{2}$ mit den Bewegungsgleichungen verträglich ist. Setzen wir diesen Wert in Gl. (5) ein, so folgt:

$$
m \dot{u}_{a}=-\frac{e}{c} f_{a, j} u_{\beta},
$$

woraus sich umgekehrt wieder $u_{c}{ }^{2}=$ const durch Integration ergibt.

Die systematische Entwicklung der Lag r a ñgeFunktion in Gl. (3) nach dem Retardierungsparameter liefert:

$$
\begin{aligned}
\int u_{c}(s) u_{c}(s-\tau) f(\sigma) d \tau=\int\left\{u_{c}^{2}-u_{c} \dot{u}_{c} \tau+\frac{1}{2} u_{c} \ddot{u}_{a} \tau^{2}+\ldots\right\} f\left(\sigma_{0}\right)+f^{\prime}\left(\sigma_{v}\right)\left[-\frac{u_{\alpha} \dot{u}_{a l}}{c^{2}} \tau^{3}\right. \\
\left.\left.+\frac{1}{c^{2}}\left(\frac{1}{3} u_{c} \ddot{u}_{c}+\frac{1}{4} \dot{u}_{a}^{2}\right) \tau^{4}\right]+\frac{1}{2} f^{\prime \prime}\left(\sigma_{0}\right)\left[-\frac{u_{a} \ddot{u}_{a}}{c^{2}} \tau^{3} \ldots\right]^{2} \ldots\right\} d \tau
\end{aligned}
$$

mit $\sigma_{0}=\frac{1}{c^{2}} u_{c^{2}} \tau^{2}$. Darin sind folgende typische Integrale enthalten:

$$
\begin{aligned}
& \int f\left(\sigma_{0}\right) \tau^{v} d \tau=\left(\frac{c}{\sqrt{-u_{C}^{2}}}\right)^{v+1} k_{v}, \\
& \int f^{\prime}\left(\sigma_{0}\right) \tau^{\prime} d \tau=\frac{v-1}{2}\left(\frac{c}{\sqrt{-u_{G}^{2}}}\right)^{v+1} k_{v^{\prime}-2}, \\
& { }^{6} \text { 1. c. }{ }^{1}, \text { I } \$ 2, \text { II } \$ 4 .
\end{aligned}
$$

$$
\int f^{\prime \prime}\left(\sigma_{0}\right) \tau^{v} d \tau=\frac{v-1}{2} \cdot \frac{v-3}{2}\left(\frac{c}{V-u_{a}^{2}}\right)^{v+1} k_{v-4},
$$

in denen die Konstanten $\boldsymbol{k}_{\boldsymbol{v}}$ durch die Integrale

$$
k_{v}=\int f\left(-\tau^{2}\right) \tau^{v} d \tau
$$

7 P. A. M. Dirac, Proc. Roy. Soc. [London] Ser. A 167, 148 [1938]. 
definiert sind. In der üblichen Näherung wird nur das Integral $k_{0}$ berücksichtigt. Gehen wir einen Schritt weiter bis zum Integral $k_{2}$ - das Integral $k_{1}$ verschwindet -, so lautet die Lag range-Funktion

$$
\mathscr{L}=-\frac{k_{0} e^{2}}{c^{3}} \sqrt{-u_{\alpha}^{2}}-\frac{3}{8} \frac{k_{2} e^{2}}{\sqrt{-u_{\gamma}^{2}}}\left[\dot{u}_{a}^{2}+\left(\frac{u_{a} \dot{u}_{\alpha}}{\sqrt{-u_{\beta}^{2}}}\right)^{2}\right]-\frac{e}{c} u_{\alpha} A_{a} .
$$

Es ist bequem, den Parameter $s$ mit der Koordinatenzeit $t$ zu identifizieren. Man vermeidet auf diese Weise die für die Diskussion lästige Nebenbedingung $u_{\alpha}{ }^{2}=-c^{2}$. Mit den Einheiten: Elektronenmasse $m_{0}=1$, Lichtgeschwindigkeit $c=1$ und Elektronenladung $e=1$ vereinfacht sich Gl. (10) folgendermaßen:

$$
\mathcal{L}=A_{0}-(\mathfrak{v}, \mathfrak{a})-k_{0} \sqrt{1-v^{2}}-\frac{3}{8} \frac{k^{2}}{\sqrt{1-v^{2}}} 3\left[\dot{\mathfrak{v}}^{2}+\left(\frac{\mathfrak{v} \dot{\mathfrak{v}}}{\sqrt{1-v^{2}}}\right)^{2}\right] .
$$

Für die neue Lagrange-Funktion ist die Abhängigkeit von der Beschleunigung charakteristisch gemäß dem Ansatz

$$
\mathcal{L}=\mathcal{L}(\mathfrak{r}, \dot{\mathfrak{r}}, \ddot{\mathfrak{r}}),
$$

der neue Freiheitsgrade mit sich bringt ${ }^{8}$. Nach E. T. Whittaker ${ }^{9}$ ergeben sich daraus Hamilton-Funktion und Hamiltonsche Gleichungen nach Einführung der zu $\dot{\mathfrak{r}}$ und $\mathfrak{v}=\dot{\mathfrak{r}}$ kanonisch konjugierten Größen

$$
\mathfrak{p}=\frac{\partial \mathscr{L}}{\partial \mathfrak{v}}-\frac{d}{d t} \frac{\partial \mathscr{L}}{\partial \dot{\mathfrak{v}}}, \mathfrak{z}=\frac{\mathrm{\partial} \mathscr{L}}{\mathrm{\partial} \dot{\mathfrak{v}}}
$$

wenn man in dem Ausdruck

$$
\mathcal{H}=\mathfrak{v} \mathfrak{p}+\dot{\mathfrak{v}} \mathfrak{z}-\mathcal{L}(\mathfrak{r}, \mathfrak{v}, \dot{\mathfrak{v}})=\mathcal{H}(\mathfrak{r}, \mathfrak{p} ; \mathfrak{v}, \mathfrak{s})
$$

für die Hamilton-Funktion $\dot{\mathfrak{v}}$ als Funktion von $\mathfrak{r}, \mathfrak{v}$ und $\mathfrak{s}$ einsetzt. Die Ha mil t o n schen Gleichungen haben die kanonische Gestalt

$$
\dot{\mathfrak{r}}=\frac{\partial \mathscr{H}}{\partial \mathfrak{p}}, \dot{\mathfrak{p}}=-\frac{\partial \mathscr{H}}{\partial \mathfrak{r}} ; \dot{\mathfrak{v}}=\frac{\partial \mathscr{H}}{\partial \dot{\mathfrak{s}}}, \dot{\mathfrak{s}}=-\frac{\partial \mathscr{H}}{\partial \mathfrak{v}} ;
$$

wie man leicht verifiziert. Es sind also $(\mathfrak{r}, \mathfrak{p})$ und $(\mathfrak{v}, \mathfrak{s})$ kanonisch konjugierte Variabelnpaare. Die Elimination ist einfacher als in dem gewöhnlichen mechanischen Problem, weil sie von der Wahl der Potentiale unabhängig ist. Aus Gl. (11) folgt:

$$
\mathfrak{s}=-\frac{3}{4} \frac{k_{2}}{\sqrt{1-v^{2}}}{ }^{3}\left(\dot{\mathfrak{v}}+\frac{(\mathfrak{v} \dot{\mathfrak{v}}) \mathfrak{v}}{1-v^{2}}\right) .
$$

Die Auflösung nach $\dot{\mathfrak{v}}$ liefert

$$
\dot{\mathfrak{v}}=-\frac{4}{3 k_{2}} \sqrt{1-v^{2}}{ }^{3}(\mathfrak{z}-(\mathfrak{v} \mathfrak{z}) \mathfrak{v}) \text {. }
$$

8 Wegen Streichung der Strahlungskraft stehen diese nicht im Widerspruch zu den Bedingungen in 1. c. ${ }^{1}$, VI.

\section{Danach ist}

$$
\begin{aligned}
\mathcal{H}=A_{0}+(\mathfrak{v}, \mathfrak{p} & +\mathfrak{a})+k_{0} \sqrt{1-v^{2}} \\
& -\frac{2}{3 k_{2}} \sqrt{1-v^{2}}\left(\mathfrak{g}^{2}-(\mathfrak{v} \mathfrak{b})^{2}\right) .
\end{aligned}
$$

Die Natur der neuen Freiheitsgrade haben wir bereits früher diskutiert ${ }^{10}$. Wenn neben der Masse noch andere Trägheitsglieder vorkommen, dann ist die Bewegung durch Anfangslage und Anfangsgeschwindigkeit noch nicht vollständig bestimmt. Der klassischen Bewegung überlagert sich eine Zitterbewegung, die durch die neuen Variabeln $\mathfrak{v}$ und $\mathfrak{g}$ beschrieben wird. Sie führt zu spinartigen Effekten, was die Erhaltungssätze sofort deutlich machen. Wie für jedes kanonische System (mit zeitunabhängiger Hamilton-Funktion) gilt der Energiesatz:

$$
E=\mathscr{H}=\text { const } .
$$

Der Drehimpulssatz, der im kugelsymmetrischen Potentialfeld ( $A_{0}=A_{0}(r)$ ) bei verschwindendem Magnetfeld $(\mathfrak{A}=0)$ gilt, hat einen von der Zitterbewegung herrührenden Zusatz:

$$
\mathfrak{M}=[\mathfrak{r}, \mathfrak{p}]+[\mathfrak{v}, \mathfrak{s}]=\text { const } .
$$

Der im kräftefreien Falle geltende Impulssatz hat die übliche Form:

$$
\mathfrak{p}=\text { const }
$$

Der Übergang zur Quantenmechanik vollzieht sich in bekannter Weise. Die kanonischen Variabeln in der Hamilton-Funktion in Gl. (18) werden zu algebraischen Größen, zwischen denen die He i s e $n$ b e r g schen Vertauschungsrelationen gel-

${ }^{9}$ Grundlehren der math. Wiss. 17, Analytische Dynamik, § 110, S. 281, Berlin 1924.

10 l. c. ${ }^{1}$, II, S. 607. 
ten. Sie lauten für die nichtkommutativen Größenphare

$$
\left[p_{\mathrm{k}}, x_{1}\right]=\frac{\hbar}{i} \delta_{\mathrm{kl}} ;\left[s_{\mathrm{k}}, v_{1}\right]=\frac{\hbar}{i} \delta_{\mathrm{kl}} .
$$

Die Übersetzung ist wegen der Nichtvertauschbarkeit der Faktoren im letzten Glied der Gl.'(18) nicht ohne weiteres eindeutig. Nach Einführung von Polarkoordinaten im Geschwindigkeitsraum gemäß $[\mathfrak{v}=v(\sin \vartheta \operatorname{eos} \varphi, \sin \vartheta \sin \varphi, \cos \vartheta)]$ erhalten wir auf Grund unsrer Betrachtungen im Anhang aus der transformierten Hamilto n-Funktion

$$
\begin{aligned}
& \mathscr{H}=-A_{0}+(\mathfrak{v}, \mathfrak{p}+\mathfrak{U})+k_{0} \sqrt{1-\mathfrak{v}^{2}} \\
& -\frac{2}{3 k_{2}}\left\{\sqrt{1-v^{2}} s_{v}^{2}+\frac{1}{v^{2}} \sqrt{1-v^{2}}{ }^{3}\left(s_{\vartheta}{ }^{2}+\frac{s_{\varphi}{ }^{2}}{\sin ^{2} \vartheta}\right)\right\}
\end{aligned}
$$

als korrespondierenden Operator den Ausdruck

$$
\begin{gathered}
\mathcal{H}=-A_{0}+(\mathfrak{v}, \mathfrak{p}+\mathfrak{U})+m \sqrt{1-v^{2}}, \\
m=k_{0}+\frac{2 \hbar^{2}}{3 k_{2}} \frac{1-v^{2}}{v^{2}}\left\{\left(1-v^{2}\right) \frac{\partial}{\partial v}\left(v^{2} \frac{\partial}{\partial v}\right)-\Lambda\right\}, \\
-\Lambda=\frac{1}{\sin \vartheta} \frac{\partial}{\partial \vartheta}\left(\sin \vartheta \frac{\partial}{\partial \vartheta}\right)+\frac{1}{\sin ^{2} \vartheta} \frac{\partial^{2}}{\partial \varphi^{2}} \cdot(24)
\end{gathered}
$$

Das Integrationselement im Geschwindigkeitsraum lautet dabei

$$
d \sigma=\frac{v^{2} \sin \vartheta d v d \vartheta d \varphi}{\sqrt{1-v^{2}}} .
$$

Durch die für die Anwendung sehr nützliche Variabelntransformation

$$
v=\mathfrak{T} \mathfrak{g} \gamma, \psi=\frac{\psi_{0}}{\mathfrak{S i n} \gamma}
$$

gehen Masseoperator und Integrationselement über in

bzw.

$$
m=k_{0}+\frac{2 \hbar^{2}}{3 k_{2}}\left\{\frac{\partial^{2}}{\partial \gamma^{2}}-1-\frac{A}{\operatorname{Sin}^{2} \gamma}\right\}
$$

$$
d \sigma_{0}=\mathfrak{C o}_{0} \gamma \sin \vartheta d \gamma d \vartheta d \varphi=\operatorname{Co} \gamma \gamma d \Omega d \gamma .
$$

Die Wellengleichung $\left\{\mathscr{H}+\frac{\hbar}{\mathrm{i}} \frac{\partial}{\partial t}\right\} \psi=0$ zeigt eine bemerkenswerte Verwandtschaft zur Dir ac schen, die besonders deutlich hervortritt, wenn wir zur Viererschreibweise zurückkehren. Es ist

mit

$$
\left\{\left(u_{\alpha}, \frac{\hbar}{i} \frac{\partial}{\partial x_{a}}+A_{a}\right)+m\right\} \psi=0
$$

$$
\begin{gathered}
m=k_{0}+\frac{\hbar^{2}}{3 k_{2}} M_{\alpha \beta}^{2}, \\
M_{\alpha \beta}^{2}=\frac{1}{i}\left(u_{\alpha} \frac{\partial}{\partial u_{\beta}}-u_{\beta} \frac{\partial}{\partial u_{\alpha}}\right) .
\end{gathered}
$$

Viererstrom und Energie-Impuls-Tensor lauten danach:

und

$$
s_{\alpha}=\int^{*} \psi^{*} u_{\alpha} \psi d U
$$

$$
T_{\alpha \beta}=\frac{1}{2} \int\left\{\psi^{*} u_{\beta} P_{\alpha} \psi+\psi u_{\beta} P_{\alpha}^{*} \psi^{*}\right\} d U .
$$

Darin bedeutet $d U$ das Integrationselement $d U=$ $d u_{1} d u_{2} d u_{3} d u_{4}$ und

$$
P_{a}=+\frac{\hbar}{i} \frac{\partial}{\partial x_{a}}+A_{a}, P_{a}^{*}=-\frac{\hbar}{i} \frac{\partial}{\partial x_{a}}+A_{\alpha} .
$$

Da die drehimpulsartigen Faktoren im Operator in Gl. (29) nur ganzzahlige Eigenwerte haben auch der von E. Schrödinger ${ }^{11}$ diskutierte Kunstgriff scheint mir nicht über diese Mannigfaltigkeit der Lösungen hinauszuführen - beschreibt die obige Wellengleichung nur Teilchen mit ganzzahligem Spin. Dem entspricht, daß es wie wir noch sehen werden - keine Übergänge zwischen Zuständen positiver und negativer Energiewerte gibt. Um so mehr fällt auf, daß die Ladungsdichte im Gegensatz zu der der Klein-Gord on schen Gleichung einen definiten Wert hat, positiv oder negativ definit, je nachdem ob es sich um einen Zustand positiver oder negativer Energie handelt.

\section{§3. Das Elektron im kräfte-und impuls- freien $\mathrm{Zustand}$}

Den einfachsten Sonderfall erhält man, wenn das äußere Feld und der Impuls des Teilchens verschwinden. Beides ist zugleich möglich, weil der Impuls im feldfreien Fall eine Konstante der Bewegung ist. Wegen der Zitterbewegung bedeutet $\mathfrak{p}=0$ im allgemeinen kein ruhendes Teilchen, das in der Quantenmechanik wegen der Unschärfebeziehung zwischen den Variabeln $\mathfrak{v}$ und $\mathfrak{s}$ gar nicht in Strenge existiert. Die Spezialisierung $\mathfrak{p}=0$ ist darum wesentlich und nicht etwa nur durch Lorentz-Transformation mit dem Falle $\mathfrak{p}=$ const verbunden. Nach dieser Vereinfachung lautet die Wellengleichung gemäß Gl. (2, 24 u. 27)

$$
\begin{gathered}
\frac{d^{2} \psi}{d \gamma^{2}}=V(\gamma) \cdot \psi \text { mit } \\
V=\frac{j(j+1)}{\operatorname{Sin}^{2} \gamma}+1-\frac{3 k_{0} k_{2}}{2 \hbar^{2}}+\frac{3 k_{2} E}{2 \hbar^{2}} \operatorname{\sigma oj} \gamma,
\end{gathered}
$$

wenn wir für den Operator $\Lambda$, der nach G! $(2,20)$ das Spinmoment darstellt $\left([\mathfrak{v}, \mathfrak{s}]^{2}=\hbar^{2} \Lambda\right)$ und in 11 Ann. Physik (5) 32, 49 [1938] (P lanck-Heft). 
unserm Falle eine Konstante der Bewegung ist, seinen Eigenwert $\Lambda=j(j+1)($ mit $j=0,1,2 \ldots)$ einsetzen.

Gl. (1) ist keineswegs trivial und nicht einmal sehr einfach. Soweit Eigenlösungen existieren, liefert die Gleichung Zustände verschiedenen Spins und bei gleichem Spin solche verschiedener Energie. Grundsätzlich führt also die Quantelung der Feldmechanik nicht nur zu einem einzigen Zustand des Elementarteilchens, sondern zu einer ganzen Folge von Zuständen, die man je nach der Lebensdauer als angeregte Zustände oder als neue Elementarteilchen ansprechen wird. Eigenwerte sind höchstens dann vorhanden, wenn das Potential $V(\gamma)$ in Gl. (1) eine Mulde bildet, deren Sohle bei negativen Werten liegt. Bereits hieraus folgt eine überraschende und bemerkenswerte Aussage über die Feldkonstanten $k_{0}$ und $k_{2}$. Es bildet sich sicher nur dann eine solche Mulde, wenn

und damit

$$
0<E<k_{0}-\frac{2 \hbar^{2}}{3 k_{2}}
$$

$$
k_{0} k_{2}>\frac{2 \hbar^{2}}{3}
$$

ist. Wegen des großen Wertes von $\hbar$ (in unsern Einheiten: $\hbar=1 / \alpha=137$ ) stellt Ungl. (4) eine sehr einschneidende Forderung dar, die nicht mit der von der L or entz schen Theorie her zu erwartenden Annahme $k_{0} \approx 1, k_{2} \leqslant 1$ verträglich ist. Die Lorentzsche Theorie kann darum nicht im üblichen Sinne als Näherung der Feldmechanik gelten. Man muß auch in nullter Näherung das Integral $k_{2}$ berücksichtigen.

Die Bedeutung dieser Ungleichungen für die Feldtheorie folgt aus der Gl. (28) in 1. c. ${ }^{1} \mathrm{~V}$ vorangehenden Relation. Danach ist

$$
k_{0}=\frac{3}{4} \int \hat{\Phi}(s) d s, \quad k_{2}=-\frac{1}{3} \int \hat{\Phi}(s) \frac{d s}{s}
$$

und $\hat{\Phi}(s)$ bedeutet die die Feldtheorie kennzeichnende Potential-Oberfunktion, aus der z.B. das statische Potential der Punktladung durch Laplace-Transformation folgt:

$$
A_{0}(r)=\frac{3}{2} \Phi\left(\frac{3 \mathrm{r}}{2}\right), \Phi(r)=\int_{0}^{\infty} \hat{\Phi}(s) e^{-\mathrm{rs}} d s .
$$

Im Falle des Coulomb-Feldes und der Maxwellschen Gleichungen ist z. B. $\hat{\Phi}=1$. Eine die Ungleichung (4) verwirklichende Oberfunktion zeigt Abb. 1. Danach ist

$$
k_{0}=\frac{3}{4} a U, \quad k_{2}=\frac{U}{3 a},
$$

und Ungl. (4) lautet

$$
k_{0} k_{2}=\frac{1}{4} U^{2}>\frac{2 \hbar^{2}}{3} .
$$

Sie läßt sich durch hinreichend große $U$ sicher erfüllen $^{12}$. Man könnte sogar $a$ so wählen, daß die klassische Bedingung $k_{0}=1$ erhalten bleibt. Aber

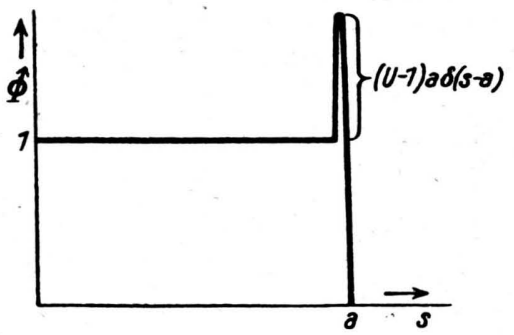

Abb. 1. Potentialoberfunktion.

in diesem Falle würde das statische Potential wegen $a \leqslant 1$ schon weit außerhalb des klassischen Elektronenradius vom Coulomb-Feld abweichen, was kaum mit den Eigenschaften der Kaskadenschauer verträglich wäre. Man sollte im Gegenteil erwarten, daß der C o u 1 o m b sche Feldverlauf noch

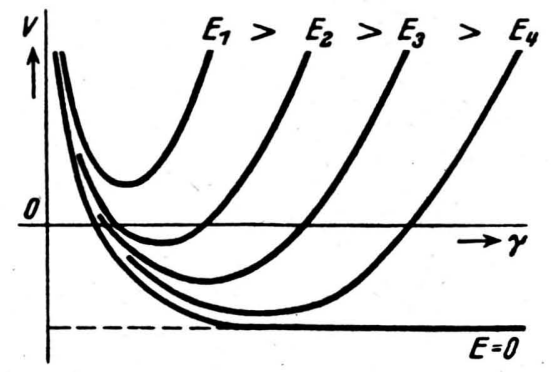

Abb. 2. Potentialverlauf in Abbhängigkeit von der Energie.

innerhalb des klassischen Elektronenradius gilt, daß also $a$ wesentlich größer sei als bisher. Trotz extremer Werte von $a$ kann die Energie klein sein. Sie ist es sicher, wenn 'sich nur $U$ hinreichend wenig von $\frac{2}{3} \sqrt{6}$ ћ unterscheidet [Ungl. (7)].

Der qualitative Verlauf des Potentials $V(\gamma)$ ist in Abb. 2 in Abhängigkeit von der Energie $E$ dargestellt. Für hinreichend große $E$ gibt es in Uber-

12 Von den Bedingungen in l. c. ${ }^{1}, \mathrm{~V}$, ist in unserm Beispiel die Photonenbedingung nicht erfüllt. Das spielt hier keine Rolle, kommt im übrigen leicht in Ordnung durch Ausglättung der Zacke. 
einstimmung mit Ungl. (2) keine Eigenlösungen. Da dasWirkungsintegral $\int \sqrt{-V} d \gamma$ zwischen den Umkehrpunkten $V(\gamma)=0$ mit abnehmendem $E$ wächst, um mit $E=0$ unendlich zu werden, muß man bei allmählich verschwindender Energie immer neue Eigenlösungen erwarten. Zum Unterschied von einem gewöhnlichen Eigenwertproblem ist die größte Energie dem kleinsten Wirkungsintegral zugeordnet. Die höheren Quantenzustände liegen energetisch tiefer, ohne daß es ein Minimum gäbe. Danach sind für die durch Gl. (1) beschriebenen Teilchen Zustände beliebig kleiner Masse möglich. Dieses Ergebnis hängt zweifellos mit unsrer Näherung zusammen. Denn die die Nebenbewegung kennzeichnende Funktion ${ }^{13}$

$$
M(p)=\frac{2 e^{2}}{m c^{3}}\left\{\frac{1}{2} k_{0}-\frac{2}{3} k_{1} p+\frac{3}{8} k_{2} p^{2}\right\}
$$

hat Nullstellen mit positivem Realteil, genügt also nicht der Bedingung der Massenstabilität. Es folgt daraus klassisch das Wessel-Diracsche Paradoxon, wonach das Teilchen bei Wechselwirkung mit äußeren Kräften unter ständigem Massenschwund und dauernder Ausstrahlung sehr rasch der Lichtgeschwindigkeit zustrebt. Quantenmechanisch setzt dieses Paradoxon in Übereinstimmung mit obigem Ergebnis ein bis zu verschwindender Masse hinreichendes Massenspektrum voraus. Natürlich kann man nicht umgekehrt schließen. Denn die Stabilität der Masse kann ebensogut durch Begrenzung des Spektrums als auch durch einschneidende Übergangsverbote erzwungen werden. Immerhin ist es bemerkenswert, daß schon ein geringfügig ansteigendes Zusatzglied im Potential der Gl. (1) genügt, um das Massenspektrum auf endlich viele Terme $\mathrm{zu}$ begrenzen.

Diese Feststellungen bedeuten, daß wir von der vorliegenden Näherung nur qualitative Aussagen erwarten dürfen. Wir dürfen uns darum mit einer groben Abschätzung für die Eigenwerte begnügen. Nach dem WBK-Verfahren erhält man letztere aus der Gleichung

$$
\frac{1}{\pi} \int_{\gamma_{1}}^{\gamma_{2}} V-V^{\prime} \cdot d \gamma=n+\frac{1}{2},\left(V^{\prime}\left(\gamma_{1}\right)=V^{\prime}\left(\gamma_{2}\right)=0\right) \text {, }
$$

wobei nach E. C. Ke mble $\mathrm{e}^{14}$ für $V^{\prime}$ wegen der Singularität im Ursprung nicht das Potential V, sondern die modifizierte Funktion

13 l. c. ${ }^{1}, \mathrm{~V}, \mathrm{Gl}$. (21).

14 The Fundamental Principles of Quantum Mechanics, London 1937, S. 107.

$$
\begin{aligned}
& V^{\prime}=\frac{\left(j+\frac{1}{2}\right)^{2}}{\operatorname{jin}^{2} \gamma}-\varkappa+\varepsilon \operatorname{cof} \gamma, \\
& \varkappa=\frac{3 k_{0} k_{2}}{2 \hbar^{2}}, \quad \varepsilon=\frac{3 k_{2} E}{2 \hbar^{2}}
\end{aligned}
$$

zu setzen ist. Näherungsweise folgt daraus

$$
\frac{1}{4}\left(\sqrt{-V^{\prime}}\right)_{\min } \cdot \Delta \gamma=n+\frac{1}{2} .
$$

$-V_{\min }^{\prime}$ bedeutet darin die Tiefe der Potentialmulde und $\Delta \gamma$ ihre Breite zwischen den Umkehrpunkten. Wenn $\varepsilon \leqslant x$ und $x \lesssim\left(j+\frac{1}{2}\right)^{2}$, d. h. wenn jedenfalls $x$ nicht groß gegen $\left(j+\frac{1}{2}\right)^{2}$ ist, erhält man

$$
\begin{gathered}
-V_{\min }^{\prime} \approx \varkappa, \\
\Delta \gamma \approx \ln (2 j+1) \frac{V^{\varkappa}}{\varepsilon}\left(\sqrt{\left.1+\frac{4}{(2 j+1)^{2}}-1\right)}\right.
\end{gathered}
$$

und nach Gl. (11)

$\varepsilon=(2 j+1) \sqrt{x}\left(\sqrt{1+\frac{4}{(2 j+1)^{2}}}-1\right) e^{-\frac{2}{\sqrt{\varkappa}}(2 \mathrm{n}+1)}$.

Setzen wir z. B.

$$
k_{0}=3535, k_{2}=1,882,
$$

so erhält man für die niedrigsten Quantenzahlen folgende Energiewerte:

$$
\begin{array}{rcc} 
& j=0 & 1 \\
1 & E=240 & 100 \\
1 & (0,417)
\end{array} .
$$

Bei der obigen Wahl der Konstanten ergibt sich also (abgesehen vom Spin) die Masse von Elektron, Meson und Y uk aw a - Quant. Doch darf man die Bedeutung dieser Utbereinstimmung nicht überschätzen. Unsere Näherung ist sicher noch weit von der Wirklichkeit entfernt. Die Frage der halbzahligen Spins ist ungeklärt und die unbegrenzte Fortsetzung des Spektrums zur Masse Null hin nicht im Einklang mit der Erfahrung. Außerdem sind Proton und Neutron noch nicht eingeordnet. Als positives Faktum dieser quantitativen Betrachtung kann man höchstens die Folgerungen aus Gl. (6) und (7) ansehen. Danach ist $U=163$ und $a=28,8$. Letzteres bedeutet, daß das CoulombFeld noch weit innerhalb des klassischen Elektronenradius besteht, was mit den bereits erwähnten Erfahrủngen über Kaskadenschauer im Einklang ist. In diesem Punkt scheint die vorliegende Näherung der L o rentz schen überlegen zu sein.

Sie ist aber weniger wegen ihrer quantitativen Ergebnisse wichtig. Ihre Bedeutung liegt m. E. 
darin, daß man an einem relativ einfachen Modell die Eigenart der gequantelten Feldmechanik und den Charakter der von ihr zu beantwortenden Fragen studieren kann. In der Tat ergeben sich Fragen über die Natur der Elementarteilchen und auch Wege zu ihrer Beantwortung, die bisher kaum ernstlich aufgegriffen werden konnten. Dies mag die Veröffentlichung der vorliegenden, quantitativ nicht befriedigenden Untersuchung rechtfertigen.

\section{Anhang: Prinzip der unveränderten Potentiale}

Der Úbergang von der klassischen $\mathrm{Hamilt}$ on Funktion zur Wellengleichung führt wegen der Nichtvertauschbarkeit der Operatoren zu einer mehrfach untersuchten Unbestimmtheit. Diese ist tiefergreifend, als es scheinen mag, wenn man von algebraischer Seite herkommt, welche die Aufmerksamkeit zunächst nur auf sehr spezielle Variationen richtet. Sie bedeutet, selbst wenn man nicht den allgemeinsten Fall vor Augen hat, daß man beliebige Potentiale verschwinden lassen und neue hervorzaubern kann. Der Hamilt on-Funktion

$$
\mathcal{H}=\frac{p^{2}}{2 m}+V(q)
$$

sind u.a. nach der gewöhnlichen Vorschrift Wellengleichungen folgender Form zugeordnet:

$$
\left\{-\frac{\hbar^{2}}{2 m} f(q) \frac{\partial}{\partial q}\left(\frac{1}{f(q)^{2}} \frac{\partial}{\partial q}\right) f(q)+V(q)\right\} \psi=E v,
$$

in denen $f(q)$ eine beliebige Funktion von $q$ sein soll. Der Ansatz ,korrespondiert" Gl. (1) und ist außerdem ,hermitesch“. Eine einfache Umformung ergibt

$$
\left\{-\frac{\hbar^{2}}{2 m}-\frac{\partial^{2}}{\partial q^{2}}+V_{1}(q)\right\} \psi=E \psi .
$$

Darin ist

$$
V_{1}=V-f\left(\frac{1}{f}\right)^{\prime \prime}
$$

entsprechend der Funktion $f$ völlig unbestimmt. Der triviale Ansatz $f=1$ führt in diesem Falle, wie man weiß, zur Übereinstimmung mit der Erfahrung.

Schon beim räumlichen Rotator ist die Lage weniger einfach. Die Hamilt o n-Funktion lautet in Polarkoordinaten

$$
\mathcal{H}=\frac{1}{r^{2}}\left\{p \vartheta^{2}+\frac{1}{\sin ^{2} \vartheta} p_{\varphi^{2}}\right\} .
$$

Hier ist der Übergang nicht durch den Ansatz $i=1$, nicht einmal durch eine einzige Funktion $f$ zu beschreiben. Der Grund dafür ist leicht zu sehen. Da das Integrationselement $d \Omega=\sin \vartheta d \vartheta d \varphi$ den Gewichtsfaktor sin $\vartheta$ enthält, ist die Bedingung für den hermiteschen Charakter abzuändern. Im übrigen liegen die Verhältnisse ähnlich wie oben.

In Gl. $(2,23)$ sind wir auf eine HamiltonFunktion der allgemeineren Form

$$
\mathcal{H}=\frac{1}{2} A(q) \cdot p^{2}
$$

gestoßen. Das Integrationselement möge $d \tau=$ $\rho(q) d q$ sein. Der zugeordnete Operator lautet allgemein:

$$
\mathcal{H}=-\hbar^{2} \frac{f}{2 \underline{Q}} \frac{\partial}{\partial q}\left(\frac{o A}{f^{2}} \frac{\partial}{\partial q}\right) f .
$$

Wie sollen wir in diesem Falle $f$ bestimmen? Die übliche Behauptung, daß darüber in jedem Einzelfalle die Erfahrung entscheiden müsse, würde bei dem Ausmaß der Unbestimmtheit darauf hinauslaufen, daß die klassischen Potentialansätze zunächst ihre Gültigkeit verlieren. Die ,trivialen“ Beispiele zeigen deutlich, daß es in Wirklichkeit nicht so ist, daß die Quantenmechanik keine neuen Potentiale schafft.

Dies legt die Vermutung nahe, daß es neben den Prinzipien der Korrespondenz und der Hermitezität ein weiteres, bisher meist angewendetes, aber noch nicht ausdrücklich formuliertes Prinzip gibt, welches die Übersetzung der klassischen Mechanik in die Quantenmechanik eindeutig macht. In Anlehnung an die trivialen Beispiele formulieren wir es versuchsweise als „Prinzip der unveränderten Potentiale“. Danach dürfen in Gl. (7) keine Glieder ohne Ableitung auftreten. Es muß also $f=1$ sein [oder einen äquivalenten Wert haben, so daß $\left(\rho A f^{\prime} / f^{2}\right)^{\prime}=o$ ist $]$; d. h. für unser Beispiel in Gl. (6) :

$$
\mathcal{H}=-\frac{\hbar^{2}}{2 \varrho} \frac{\partial}{\partial q}\left(\varrho A \frac{\partial}{\partial q}\right) .
$$

- Der Ableitung des Operators in Gl. $(2,24)$ wurde dieses Prinzip zugrundegelegt unter Benutzung des Integrationselementes aus Gl. $(2,25)$. Letzteres ergibt sich aus der Forderung, daß $H d \sigma$, nach Gl. $(2,23)$ also z. B. speziell $\sqrt{1-v^{2}} d \sigma$, L o r e n t zinvariant sein muf. 